\title{
REBEn \\ Erros de medicação em pediatria: análise da documentação de enfermagem no prontuário do paciente
}

\author{
Medication errors in pediatrics: analysis of nursing records on the patient chart
}

Errores de la medicación en pediatría: analisis de los documientos de enfermería en los documientos hospitalares de los pacientes

Liliane Rodrigues Melo

Aluna do $3^{\circ}$ ano de Graduação em Enfermagem do Departamento de Enfermagem da UNIFESP, bolsista de Iniciação Científica do CNPq.

Mavilde Luz Gonçalves Pedreira

Enfermeira, Doutora em Enfermagem, Professora Adjunto do Departamento de Enfermagem da UNIFESP-EPM. Autor correspondente. E-mail: mavilde@denf.epm.br

Pesquisa realizada com fomento concedido pelo Conselho Nacional de Desenvolvimento Científico e Tecnológico CNPq. Trabalho premiado - Melhor Pesquisa Científica Expo-Enf 2004, Departamento de Enfermagem da UNIFESP-

\section{RESUMO}

Estudo descritivo e transversal realizado em três unidades pediátricas de um hospital universitário que objetivou, por meio da análise do prontuário do paciente, identificar erros de medicação. Estes erros podem caracterizarse como falhas no registro do processo de atendimento, que não chegaram a comprometer a segurança dos pacientes. Nos 68 prontuários investigados identificaram-se 1717 erros de registro, compondo 21,1\% das 8152 doses de medicamentos ou soluções prescritas no período investigado. Foram categorizados mais de 13 tipos de erros, destacando-se os de omissão de dose ou de registro da execução da dose $(75,7 \%)$. Frente aos eventos verificados, sugere-se o desenvolvimento de programas de educação continuada e a criação de ferramentas de gestão da assistência que permitam desenvolver esta prática, monitorando resultados obtidos. Descritores: Enfermagem pediátrica; Erros de medicação; Uso de medicamentos; Gerenciamento de segurança; Registros de enfermagem.

\section{ABSTRACT}

Descriptive and correlational study which aimed to verify medication errors through the analyzes of medical charts of children admitted in three pediatric wards of a university hospital. The errors could represent record gaps verified on the care process documentation, that do not compromise the patient safety. In the 68 medical charts 1717 errors were verified, $21.1 \%$ of the 8152 drugs doses or solutions ordered during the studied period. More than 13 categories of errors were identified, omission errors (dose or records) were the most frequents (75.7\%). The types of errors indicated the need of continuous education and the implementation of management tools that allowed the development of the practice and monitoring results.

Descriptors: Pediatric nursing; Medication errors; Drugs utilization; Safety management; Nursing records.

\section{RESUMEN}

Estudio descriptivo y correlacional realizado en unidades pediátricas de un hospital de enseñanza por la analize de errores de la medicación en los prontuarios de pacientes, que podrían también caracterizar errores de documentación, imperfecciones en lo registro del proceso del cuidado que puderan no comprometer la seguridad de los paciente. En los 68 prontuarios 1717 errores fueron verificados en $21,1 \%$ de las 8152 dosis de medicamientos o soluciones prescrebidas durante el período estudiado. Más de 13 tipos de errores fueron categorizados, en la mayoría (75,7\%) los errores de omisión (dose ò registro). Los resultados indicaron la necesidad de programas de educación y de uso de herramientas de gerencia que permitan el desarrollo de la práctica y acompañamiento de los resultados.

Descriptores: Enfermería pediátrica; Errores de la medicación; Utilización de medicamentos; Administración de la seguridad; Registros de enfermería.

Melo LR, Pedreira MLG. Erros de medicação em pediatria: análise da documentação de enfermagem no prontuário do paciente. Rev Bras Enferm 2005 mar-abr; 58(2):180-5.

\section{INTRODUÇÃO}

Em publicação editada pelo Institute of Medicine dos Estados Unidos da América, em 2000, intitulada "Errar é Humano: Construindo um Sistema de Saúde mais Seguro", um grupo de especialistas norteamericanos analisou e discutiu os principais componentes do sistema de saúde, capazes de conduzir a erros e dentre estes, problemas relacionados ao uso de medicamentos destacam-se como freqüentes e potencialmente fatais ${ }^{(1)}$.

Relatam que, a ocorrência de erros é inerente ao processo cognitivo humano e, neste contexto, profissionais de saúde são passíveis de cometê-los. Esforços devem ser implementados na tentativa conhecer e explorar tais eventos, com a finalidade de desenvolver estratégias que conduzam a prevenção e conseqüentemente promovam a segurança do paciente ${ }^{(2)}$. Este assunto caracteriza-se como de extrema 
relevância pois, os erros relacionados a técnica e procedimentos na área da saúde, podem resultar em tragédia para pacientes e suas famílias, aumentar o tempo de internação e os custos hospitalares. Adicionalmente, podem ter um efeito dramático na vida de profissionais de saúde dedicados e envolvidos na assistência ${ }^{(3)}$.

Estima-se que anualmente cerca de 44.000 a 98.000 norte-americanos morram devido a erros cometidos durante 0 atendimento à saúde, compondo a oitava causa de mortalidade nesse país ${ }^{(1,2)}$. Outros estudos demonstram que a cada ano cerca de um milhão de pacientes são vítimas de eventos adversos com fármacos e destes, 140.000 morrem. A probabilidade de morte decorrente de evento adverso com fármacos em pacientes hospitalizados (390/dia) é três vezes maior, do que as decorrentes de acidentes automobilísticos (125/dia) ${ }^{(4)}$.

A dimensão desta problemática ultrapassa a premissa central de prejuízo para o paciente envolvido, acarretando repercussões para o profissional e instituição, bem como, outras de caráter social, ético, econômico e cultural. A fim de prevenir erros de medicação, devem ser implementados sistemas e métodos de trabalho que considerem a segurança do paciente. Na década de sessenta do século passado, teve início na prática de enfermagem a avaliação dos cinco certos: droga, dose, via, hora e paciente certos. Na década de noventa surgiu a idéia de incluir-se mais um certo: a documentação correta ${ }^{(5-7)}$.

Deste modo, faz-se importante incluir na documentação de enfermagem o planejamento da administração da terapia medicamentosa, a fim de prevenir eventos adversos e erros de medicação. Salienta-se a necessidade de padronização nas instituições de saúde de nomenclaturas, símbolos e métodos de anotação do processo de consecução da terapia.

Um erro de medicação pode ser definido como "qualquer evento evitável que pode ser causado ou surgir do uso inconveniente ou falta de uma medicação, ou causar prejuízo (dano ou injúria) ao paciente, enquanto a medicação está sob o controle dos profissionais da saúde, pacientes ou consumidores; tais eventos podem estar relacionados à prática profissional, aos produtos para o cuidado à saúde, procedimentos e sistemas, incluindo a prescrição, comunicação da prescrição, rótulo do produto, embalagem e nomenclatura; a composição, a distribuição, a administração, a educação dos profissionais e pacientes; a supervisão e 0 uso"(5).

Um erro pode ser definido como a falha de uma ação planejada para ser realizada a contento, ou o uso de um plano errado para executar uma ação(6). Os erros de medicação podem estar relacionados à falha na implementação dos cinco certos ${ }^{(7)}$. Podem também decorrer de falhas no planejamento do aprazamento da prescrição médica, acarretando incompatibilidade entre os medicamentos administrados. Outras possíveis categorias de erros são: omissão da dose (não realização da medicação), administração de dose errada, atraso na administração, não orientação do paciente, falta de monitorização, dentre outros ${ }^{(8)}$.

Todas as atividades de enfermagem executadas junto ao paciente, do planejamento ao acompanhamento dos resultados dos cuidados de enfermagem, devem ser registradas de modo sistematizado em todas as unidades de atendimento hospitalar, incluindo aspectos relativos a terapia medicamentosa, explicitados nas intervenções de enfermagem propostas, a partir de diagnósticos ou problemas de enfermagem identificados pelo enfermeiro. Posteriormente, é atribuição desse mesmo profissional o acompanhamento e registro dos resultados obtidos frente às intervenções propostas. Fazem ainda parte dos documentos de enfermagem as anotações, executadas por todos os membros da equipe de enfermagem.

Assim, para a implementação segura da terapia medicamentosa, enfermeiros devem ter conhecimentos sobre o correto preparo e administração de fármacos e soluções. A partir da prescrição médica, executam 0 aprazamento da administração, prescrevem cuidados concernentes a administração e monitorização, conforme o tipo de terapêutica, e registram a resposta do paciente ao tratamento, incluindo possíveis eventos adversos relacionados ao uso de fármacos e soluções.

Por meio da análise do prontuário do paciente, registro legal de todas as ações da equipe de saúde implementadas durante 0 atendimento, esta pesquisa teve como objetivo identificar erros de medicação, podendo estes, devido ao tipo de estudo implementado, configurarem erros propriamente ditos ou ainda, representarem erros na documentação, caracterizados por falhas cometidas durante o registro do processo de atendimento mas, que podem não ter comprometido a segurança dos pacientes submetidos a terapia medicamentosa. Pretende-se com estes achados, analisar os registros do processo de trabalho da enfermagem, quanto ao planejamento e execução da terapêutica medicamentosa prescrita pela equipe médica, identificando questões que necessitem melhorias com enfoque na prevenção de erros de medicação e na segurança do paciente.

\section{MÉTODO}

Estudo descritivo e transversal realizado a partir de dados coletados de prontuários de crianças internadas em três unidades pediátricas, cirurgia pediátrica, infectologia pediátrica e cuidados intensivos pediátricos, de um hospital universitário do município de São Paulo, após consentimento da chefia de enfermagem da instituição para realização da pesquisa. As unidades investigadas realizam atendimento à crianças da faixa etária neonatal, pediátrica e adolescência, sendo que, a unidade de cirurgia pediátrica conta com nove leitos, a infectologia pediátrica com 11 e a de cuidados intensivos com nove leitos.

Planejou-se que a população do estudo seria composta pelos documentos de enfermagem e prescrições médicas contidos nos prontuários de todas as crianças internadas nas unidades pediátricas investigadas, no mês de outubro de 2003. Entretanto, devido a impossibilidade de acesso a alguns prontuários ou documentos, das 79 crianças internadas nas unidades investigadas, durante o período de estudo, fizeram parte da amostra, documentos constantes nos prontuários de $68(86,1 \%)$ crianças.

A coleta de dados foi realizada por um dos pesquisadores após aprovação da pesquisa pelo Comitê de Ética em Pesquisa da Instituição, conforme parecer $n^{0} 0321 / 03$. Para a coleta dos dados foi utilizado um instrumento, denominado Protocolo, composto pelas variáveis estudadas, tais como: a identificação da unidade, do paciente, da terapia medicamentosa prescrita, do aprazamento da prescrição médica, dos registros de execução da prescrição médica, bem como, todos os diagnósticos, prescrições, evoluções e anotações de enfermagem referentes ao planejamento e implementação da terapia medicamentosa.

A análise dos dados foi implementada com base na natureza das variáveis investigadas nesta pesquisa, apresentando-se as variáveis categóricas, segundo freqüências absoluta e relativa, e as numéricas contínuas, segundo mediana, média e desvio padrão. Posteriormente, para a classificação dos erros de medicação foi utilizado como referencial teórico a Taxonomia proposta pelo National Coordinating Council for Medication Error Reporting and Prevention (NCC MERP) de $1998^{(9)}$.

\section{RESULTADOS}

Foram analisados os prontuários de 68 crianças internadas nas unidades de infectologia pediátrica, cirurgia pediátrica e cuidados intensivos pediátricos (UCIP) estando na Tabela 1 apresentados 0 número, a idade e o tipo de diagnóstico médico das crianças, segundo a unidade de internação.

Conforme dados da Tabela 1, observa-se maior proporção (45,6\%) de crianças internadas na unidade de infectologia durante o período de estudo. Quanto a idade identificou-se predomínio de crianças na faixa etária pré-escolar e lactente, sendo que crianças com diagnósticos clínicos predominaram nas unidades de infectologia e cuidados intensivos pediátricos, respectivamente $100,0 \%$ e $72,0 \%$, enquanto conforme o esperado, os diagnósticos cirúrgicos foram predominantes 
(95,0\%) na unidade de cirurgia pediátrica.

Tabela 1. Número, idade (anos) e tipo de diagnóstico médico de crianças, segundo unidade de internação pediátrica. São Paulo, 2003.

\begin{tabular}{lccccccc}
\hline \multirow{2}{*}{$\begin{array}{l}\text { Variaveis relativas } \\
\text { as crianças }\end{array}$} & \multicolumn{2}{c}{ Infecto } & \multicolumn{2}{c}{ CirPed } & \multicolumn{2}{c}{ UCIP } \\
\cline { 2 - 8 } & $\mathbf{n}$ & $\%$ & $\mathbf{n}$ & $\%$ & $\mathbf{n}$ & $\%$ \\
\cline { 2 - 8 } & 31 & 45,6 & 19 & 27,9 & 18 & 26,5 \\
Num. de crianças & Diagnostico Medico & & & & & & \\
Cínico & 31 & 100 & 1 & 5,3 & 13 & 72,2 \\
Cirúrgico & - & - & 18 & 94,7 & $\mathbf{5}$ & 27,8 \\
Idade (media e DP) & $3,8 \pm 3,75$ & & $5,1 \pm 3,6$ & \multicolumn{2}{c}{$1,9 \pm 5,1$} \\
\hline
\end{tabular}

Nos prontuários destas 68 crianças foram identificadas 608 prescrições médicas. Quanto aos documentos da enfermagem, totalizaram $590(97,0 \%)$, pois $18(3,0 \%)$ documentos não foram encontrados no prontuário dos pacientes. O número de diagnósticos, prescrições, anotações e evoluções de enfermagem identificados nos prontuários, segundo a unidade investigada, encontra-se descrito na Figura 1.

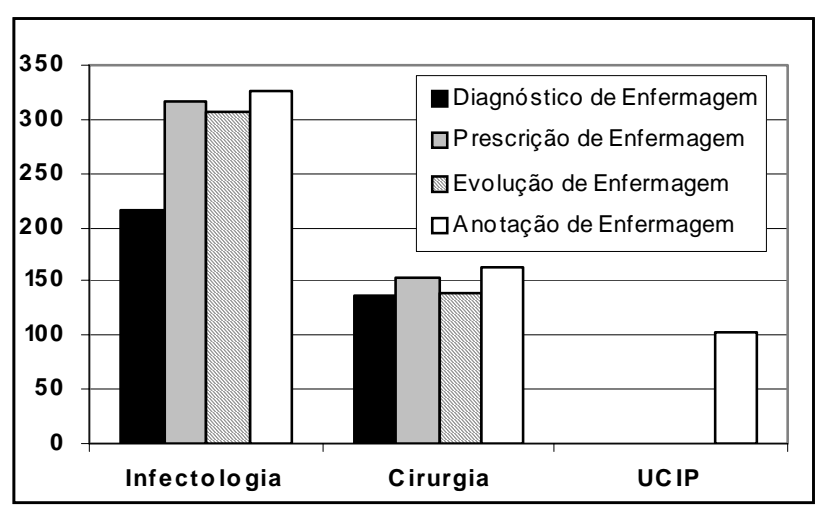

Figura 1. Número e tipo de registros de enfermagem. São Paulo, 2003.

Pode-se observar na Figura 1 que na UCIP foram identificadas quase que exclusivamente as anotações de enfermagem nos 104 documentos analisados, enquanto nas demais unidades investigadas, apesar de observarem-se variações, todas as fases do processo de enfermagem apresentaram registros nos 340 e 164 documentos investigados, respectivamente das unidades de infectologia e cirurgia pediátrica. Verifica-se que a unidade de cirurgia pediátrica apresentou registros mais completos da sistematização da assistência de enfermagem.

Nas 608 prescrições médicas analisadas foram identificados 168 diferentes tipos de medicamentos e soluções, perfazendo um total de 8152 doses prescritas de fármacos, soluções e hemocomponentes. $\mathrm{Na}$ Tabela 2 são identificados os tipos de administração dos fármacos e soluções, segundo as categorias intermitente e contínua, destacandose a unidade de infectologia pediátrica como a que apresentou maior número de doses prescritas de fármacos, soluções e hemocomponentes. Das 7633 (100,0\%) doses de medicamentos de administração intermitente, maior proporção $(52,4 \%)$ foi identificada na unidade de infectologia pediátrica e das $513(100,0 \%)$ administrações contínuas, a
UCIP apresentou maior proporção (60,8\%). Observa-se deste modo, maior emprego, em todas as unidades da administração intermitente da terapia medicamentosa.

A classificação dos fármacos e soluções apontou 29 categorias, destacando-se os antimicrobianos (29,8\%), antitérmicos (14,7\%), analgésicos opióides e sedativos (12,6\%), broncodilatadores $(7,8 \%)$, antieméticos - espasmódicos (5,9\%), descongestionantes nasais $(4,2 \%)$, anti-hipertensivos (3,9\%), corticóides (3,4\%), analgésicos $(2,8 \%)$, antiácidos $(2,6 \%)$, vitaminas $(2,2 \%)$, drogas vasoativas $(2,3 \%)$, diuréticos $(1,5 \%)$, laxativos $(1,4 \%)$, anticonvulsivantes $(1,4 \%)$, antiinflamatórios $(0,9 \%)$, anticoagulantes $(0,7 \%)$, anti-histamínicos $(0,5 \%)$ e outros $(1,4 \%)$. Quanto as soluções, destacaram-se os do tipo soro de manutenção (72,8\%), soro de reposição (12,8\%), nutrição parenteral total $(8,5 \%)$ e soro de correção (5,94\%). Foram identificadas administrações de hemocomponentes em todas as unidades investigadas, perfazendo total de 79 infusões, das quais $93,7 \%$ foram realizadas na UCIP, $5,1 \%$ na infectologia pediátrica e 1,2\% na cirurgia pediátrica.

No período de estudo foram computados 8152 registros de doses de fármacos ou soluções prescritas e em 1717 (21,1\%) verificaram-se erros. Ressalta-se que algumas destas falhas relacionaram-se a erros na documentação, sendo elaborada uma classificação, segundo a tipologia relacionada destacando-se que, devido ao tipo de estudo realizado, a análise documental, os erros de medicação identificados podem, ou não, ser entendidos como eventos adversos ao paciente.

Tabela 3. Tipo de erros de medicação em unidades pediátricas de um hospital universitário da cidade de São Paulo, 2003.

\begin{tabular}{|c|c|c|}
\hline Tipos de erros de medicaçấ & n & $\%$ \\
\hline $\begin{array}{l}\text { Omisss̃o da dose ou registro de execuçäo da } \\
\text { dose }\end{array}$ & 1299 & 75,7 \\
\hline $\begin{array}{l}\text { Medicaçâo prescrita pelo médico e registrada } \\
\text { como suspensa pela enfermagem }\end{array}$ & 175 & 10,3 \\
\hline $\begin{array}{l}\text { Medicação na administrada por fata do } \\
\text { medicamento na farmácia }\end{array}$ & 119 & 6,9 \\
\hline Hora errada & 40 & 2,4 \\
\hline Droga errada & 40 & 2,4 \\
\hline Medicação preparada, mas nüo checada & 7 & 0,5 \\
\hline $\begin{array}{l}\text { Medicaçâo prescrita e ns̃o administrada, por } \\
\text { fatta de via de acesso }\end{array}$ & 6 & 0,3 \\
\hline Via ns̃o identificada & 6 & 0,3 \\
\hline Velocidade enrada & 5 & 0,2 \\
\hline Administroçăo de medicamento nâo autorizada & 4 & 0,2 \\
\hline Via emada & 4 & 0,2 \\
\hline Outros & 6 & 0,3 \\
\hline Total & 1717 & 100,0 \\
\hline
\end{tabular}

Conforme se nota na Tabela 3 foram identificados mais de 13 diferentes tipos de erros de medicação, sendo que, alguns constituíram

Tabela 2. Tipo e número de prescrições de fármacos, soluções e hemocomponentes, segundo unidade pediátrica. São Paulo, 2003.

\begin{tabular}{|c|c|c|c|c|c|c|}
\hline \multirow[t]{2}{*}{ Tipo de administraçao } & \multicolumn{2}{|c|}{ Infectologia } & \multicolumn{2}{|c|}{ Cirurgia Pediatrica } & \multicolumn{2}{|c|}{ UCIP } \\
\hline & $\mathbf{n}$ & $\%$ & $\mathbf{n}$ & $\%$ & n & $\%$ \\
\hline $\begin{array}{l}\text { Intermitente } \\
\text { Continus }\end{array}$ & $\begin{array}{c}4002 \\
86\end{array}$ & $\begin{array}{c}97,8 \\
2,2\end{array}$ & $\begin{array}{c}1671 \\
115\end{array}$ & $\begin{array}{l}93,6 \\
6,4\end{array}$ & $\begin{array}{c}1960 \\
312\end{array}$ & $\begin{array}{l}86,3 \\
13,7\end{array}$ \\
\hline Total & 4088 & 100,0 & 1972 & 100,0 & 2271 & 100,0 \\
\hline
\end{tabular}


falhas na documentação. Amplamente destacados encontram-se os erros de omissão, perfazendo $75,7 \%$ do total de falhas identificadas. Erros de omissão correspondem à não administração de uma dose prescrita do medicamento ou o não registro da execução da medicação pela enfermagem.

Identificaram-se $175(10,3 \%)$ erros nos quais a medicação prescrita pelo médico foi registrada como suspensa pela enfermagem, evidenciando provavelmente falha no registro de ordens verbais feitas pela equipe médica. Este tipo de erro de documentação é identificado quando a enfermeira suspende a administração de uma dose da medicação, utilizando rotineiramente 0 ato de "circular" o horário designado para a administração, sendo que, quando a equipe médica não documentou a suspensão da medicação, o erro de registro foi encontrado.

Detectaram-se 119 (6,9\%) situações nas quais a medicação fo suspensa pelo médico e não suspensa pela enfermagem, configurando provavelmente um erro de documentação que deve ser prevenido por poder acarretar a administração de uma medicação suspensa.

Medicamentos prescritos pela equipe médica não foram administrados por falta do medicamento na farmácia em $40(2,4 \%)$ doses analisadas, configurando erro de medicação que pode comprometer a eficácia da terapia planejada e acarretar prejuízo à segurança do paciente. Erros de horário, denominados hora errada foram verificados em $40(2,4 \%)$ situações, nas quais a medicação foi executada fora do intervalo de tempo planejado para a administração. Consideraram-se erros de horário aqueles que ultrapassaram 30 minutos, para mais ou para menos, dos horários pré-estabelecidos no aprazamento da prescrição médica. Os outros $44(2,3 \%)$ tipos de erros identificados podem constituir erros de medicação ou de documentação do processo de execução da terapia medicamentosa pela enfermagem.

Adicionalmente investigou-se nos documentos de enfermagem a presença de registros referentes à terapia medicamentosa, conforme dados apresentados na Figura 2. Os diagnósticos de enfermagem não foram submetidos a este tipo de análise, devido a necessidade de conhecer as características definidoras a fim de relaciona-los à terapia medicamentosa, dado não constante na documentação investigada.

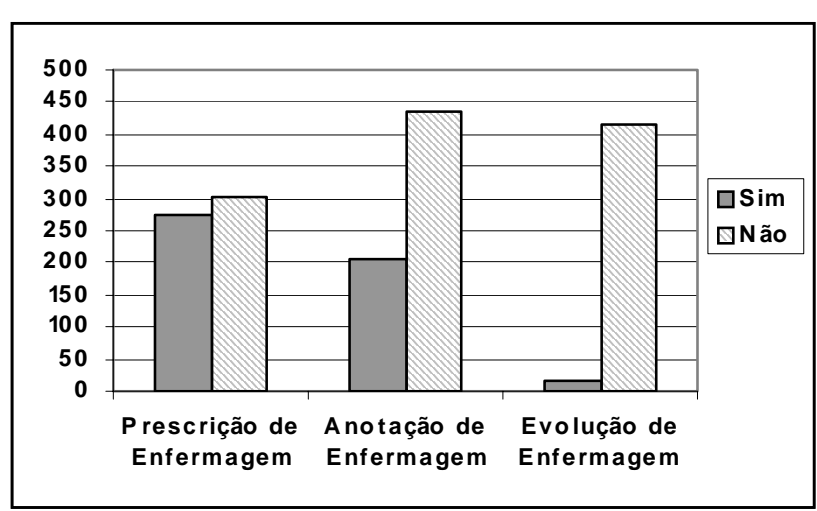

Figura 2. Presença de registros referentes a terapia medicamentosa nos documentos de enfermagem. São Paulo, 2003

A figura acima mostra que a equipe de enfermagem realiza registros referentes à terapia medicamentosa, sendo que, divergem conforme a fase do processo de sistematização da assistência de enfermagem. Foram mais presentes nas prescrições de enfermagem (274) e nas anotações de enfermagem (204), sendo a menor freqüência identificada nas evoluções de enfermagem (15).

Segundo os dados primários, estes registros têm relação com a prática da execução da terapia, como, por exemplo, controle de infusão, manutenção de cateteres, balanço hídrico, registros de início da terapia de transfusão, instalação de soluções, início e término de terapias, dentre outros.

\section{DISCUSSÃO}

Nas unidades hospitalares pesquisadas é utilizado o sistema manual de registros dos cuidados prestados ao paciente, constituindo fonte de informações de todas as atividades realizadas pela equipe, permitindo identificar a assistência implementada e medir os resultados obtidos. É possível estimar que a enfermagem seja responsável por mais de 50\% das informações contidas no prontuário do paciente, não só por dados fornecidos por enfermeiros, mas também por técnicos e auxiliares de enfermagem ${ }^{(10,11)}$.

O registro das ações de enfermagem no prontuário é um instrumento de grande significado na assistência de enfermagem, sendo indispensável para a adequada prestação do cuidado ao paciente ${ }^{(12)}$. Em virtude desta realidade nota-se a necessidade de melhorar este processo nas unidades pediátricas analisadas, pelo fato de não se encontrar a totalidade dos documentos de enfermagem e em outros identificarem-se lacunas no processo de enfermagem, destacando-se a UCIP onde se realiza apenas a anotação de enfermagem. Vale destacar que, nessa unidade existe uma ficha de monitorização e controle das funções vitais dos pacientes. Apesar de fornecer inúmeros indicadores das condições clínicas das crianças e dos cuidados de enfermagem realizados, não substitui as outras fases do processo de enfermagem, que contribuem para a identificação dos resultados e conseqüentemente da qualidade dos cuidados de enfermagem desenvolvidos.

Dentre as informações relacionadas a terapia medicamentosa, conforme a fase do processo de enfermagem, notamos um déficit ainda maior conforme a Figura 2, na qual se identifica a ausência marcante de dados ou informações sobre a terapia, eminentemente nas evoluções de enfermagem. Frente a diversidade de cuidados de enfermagem relacionados a execução da terapia medicamentosa, aos avanços tecnológicos na área e as características específicas de crianças submetidas a este tipo de tratamento, faz-se importante mudar a realidade observada para que se possa obter maiores informações que comprovem adequado monitoramento do paciente quanto aos resultados obtidos com a implementação da terapêutica prescrita pela equipe médica. Observase que a prática de enfermagem evolui constantemente, técnica e cientificamente, mas apresenta um grande desafio, realizar práticas fundamentadas em evidências científicas e produzir novos conhecimentos a partir da experiência vivenciada no cotidiano assistencial, sendo a documentação desta prática que permite tais constatações.

Dentre as mais de 29 classificações de medicamentos analisados foram encontrados 168 tipos de diferentes fármacos prescritos nas três unidades pediátricas analisadas, sendo que, os antimicrobianos foram os fármacos mais freqüentemente verificados. Estes resultados revelam a diversidade de conhecimentos que a equipe multiprofissional necessita possuir para a implementação segura da terapia medicamentosa.

Quanto ao modo de administração identificou-se maior emprego do tipo intermitente, tendo este relação com a finalidade terapêutica do fármaco ou solução prescrita, podendo constituir administrações imediatas ou as infusões realizadas em intervalo de tempo inferior a duas horas. Calculou-se que cada uma das 68 crianças recebeu durante trinta dias, em média, mais de 112 doses de fármacos ou soluções administrados de modo intermitente, demonstrando que na instituição investigada a terapia medicamentosa caracteriza-se como parte importante das atribuições da enfermagem. Segundo survey realizado pela Academia Americana de Enfermagem no planejamento, execução e avaliação da assistência de enfermagem prestada ao paciente hospitalizado e em situação de emergência, aproximadamente dois terços do tempo de trabalho de enfermeiros são destinados à implementação dessa terapia ${ }^{(13)}$.

Todas as 513 administrações de modo contínuo foram de fármacos e soluções intravenosos, segundo os dados primários. Cabe salientar que, caracterizam-se como tipos contínuos de administração, aqueles 
que ultrapassam duas horas de infusão, sendo necessária a instituição de cuidados que promovam a monitorização da aceitação do paciente ao tratamento instituído, requerendo por vezes, conhecimentos especializados da enfermagem para sua execução ${ }^{(14)}$.

Quanto a administração de hemocomponentes alguns passos devem sempre ser executados, sendo estes a checagem da prescrição médica, a seleção de materiais e equipamentos de infusão, o preparo do paciente, a obtenção do hemocomponente no banco de sangue, o preparo para administração, o início da infusão, a monitorização da infusão e suspensão em casos de aparecimento de reações adversas ${ }^{(14)}$. Para a consecução segura da terapia de transfusão, vários cuidados são necessários, utilizando-se em muitas instituições de saúde a prática da dupla checagem, não identificada nos prontuários analisados. A dupla checagem refere-se ao processo de trabalho no qual duas pessoas estão envolvidas na execução da atividade afim.

Grande proporção de erros de medicação foi identificada nos prontuários analisados, sendo estes relacionados a erros de execução ou de documentação da terapia medicamentosa. Identificou-se nesta pesquisa, mais de 13 diferentes classificações de erros, sendo que divergem quanto ao tipo de erro de medicação, constituindo situações que comprometeram a segurança do paciente, que potencialmente poderiam ter comprometido, ou que não atingiram ao paciente, mas, foram decorrentes de falhas na documentação deste processo assistencial pela enfermagem.

Destacaram-se os erros de omissão, perfazendo a grande maioria $(75,7 \%)$ das falhas identificadas, resultado verificado em pesquisa realizada em 36 hospitais norte-americanos sobre a temática, como 0 segundo tipo de erro de medicação identificado ${ }^{(15)}$. Apesar de acreditarmos que grande parte destes erros de medicação refere-se a falhas de checagem do procedimento, observa-se necessidade de intervir no processo assistencial da enfermagem de modo a garantir a melhora desta fase de registro da terapia medicamentosa.

Profissionais de enfermagem que atuam na assistência e gestores de instituições de saúde devem desenvolver metodologias de trabalho que sejam orientadas à prevenção de eventos adversos, sendo 0 planejamento e os registros hospitalares as principais formas de mensuração dos resultados obtidos.

Em estudo realizado por Bohomol(16) no município de São Paulo identificou-se que profissionais de enfermagem não atribuíam falhas a situações que configuravam erros de omissão. Outro estudo realizado em Londrina, também verificou o desconhecimento da equipe de enfermagem sobre vários erros de medicação, concluindo que são necessários programas de educação continuada sobre a temática em hospitais, a fim de contribuir com subsídios que promovam a segurança dos pacientes submetidos a terapia medicamentosa ${ }^{(17)}$.

Os erros de documentação identificados, sob o aspecto legal da prática profissional, poderiam gerar processos judiciais. Esta falta de respaldo da prática realizada, passa a comprometer a segurança do próprio profissional ou da instituição de saúde. Quanto à dimensão desta problemática em instituições de saúde norte-americanas, estudos apontam que cada paciente hospitalizado está sujeito a ocorrência de, em média, dois erros por dia, com aumento do tempo de internação em até 4,6 dias, estimando-se ainda que, 2,0\% dos pacientes hospitalizados anualmente sejam vítimas de erros de medicação, com custo adicional em cada erro de cerca de cinco mil dólares no tratamento(1).

Sabe-se que crianças estão sujeitas a maior proporção de erros devido ao uso de doses de fármacos fracionadas, sendo descrita por Konh $^{(1)}$ em 2000, proporção de 4,9 e 4,5 erros de prescrição em cada 1000 prescrições médicas investigadas em dois hospitais. Torna-se difícil a comparação destes resultados com os obtidos nesta pesquisa pois, a partir da prescrição médica, várias são as atribuições de enfermagem na execução da terapia, e sabe-se que quanto maior o número de tarefas a serem executadas, maior a probabilidade da ocorrência de falhas e que, quanto mais complexa for a atividade desenvolvida maior a necessidade de preparo do profissional para reduzir a chance de erros. Adicionalmente a melhor, maior, mais preparada e dedicada força de trabalho ainda poderá cometer erros, pois esta falibilidade é uma parte imutável da natureza humana. Entretanto, esta falibilidade pode ser agravada quando as práticas, procedimentos, protocolos, rotinas, técnicas e equipamentos utilizados pelos trabalhadores forem inadequados, complexos e por si só inseguros ${ }^{(18)}$.

Deste modo, uma visão sistêmica de prevenção e análise de erros na medicação deve ser implementada, sendo por vezes necessária a total reestruturação dos processos de trabalho para o alcance dos resultados esperados. Estes processos de trabalho estão inseridos dentro de um contexto maior, o sistema de atendimento e, todo o sistema deve ser considerado como perfeitamente desenhado para produzir os seus próprios resultados ${ }^{(19,20)}$. Ou seja, a solução é aprender com os erros, não por meio de analises individuais e sim, redesenhando 0 sistema para reduzir a presença dos mesmos e criando uma cultura de segurança que permita a contínua possibilidade de gerenciar riscos ${ }^{(21)}$.

Certas premissas devem ser seguidas para criar uma cultura de segurança, destacando-se o equilíbrio entre a eficiência e a real possibilidade produtiva, a criação e a sustentação da confiança na organização, a gestão ativa e participativa nos processos de mudança, a participação dos profissionais nas decisões de desenho dos processos de trabalho e a implementação de cultura de aprendizado constante nas questões vinculadas ao processo de trabalho ${ }^{(18,19)}$.

Para que a enfermagem consiga controlar o processo de medicação sob sua responsabilidade, garantindo a segurança do paciente, fazemse necessários programas de educação continuada e incorporação de tecnologias e sistemas de informação na prática assistencial(10). A partir da prescrição médica, na maioria das instituições de saúde, a equipe de enfermagem é responsável pelo preparo e administração da terapia, caracterizando-se como executantes de maior número de procedimentos para a realização. A implementação de prescrições informatizadas, uso de código de barras e de bombas de infusão inteligentes são estratégias capazes de reduzir os erros de medicação relacionados a prática da enfermagem pois, envolvem suporte para as ações de planejamento e se estendem até a implementação da terapia medicamentosa no paciente ${ }^{(22,23)}$.

É importante que o enfermeiro conheça as responsabilidades legais que envolvem a execução da terapia medicamentosa para promover a segurança do paciente e realizar cuidados competentes e adequados as necessidades do paciente e as características da terapia prescrita ${ }^{(24)}$.

\section{CONCLUSÕES}

Foram identificados 1717 erros, podendo estes constituir erros de medicação ou de documentação de enfermagem, nos 68 prontuários de crianças investigados. Estes erros estiveram presentes em 21,1\% das 8152 doses de medicamentos ou soluções administradas no período de estudo.

Mais de 13 tipos de erros de medicação ou de documentação de enfermagem foram categorizados, destacando-se os erros de omissão (de dose ou de registro da execução da dose) como os mais freqüentes $(75,7 \%)$ e a partir destes resultados considerou-se como principal estratégia para a mudança da realidade identificada o desenvolvimento de programas de educação continuada, bem como, a criação de ferramentas de gestão da assistência que permitam desenvolver a prática e monitorar os resultados obtidos. 


\section{REFERÊNCIAS}

1. Konh LT, Corrigan JM, Donaldson MS. A comprehensive approach to improving patient safety. In: Konh LT, Corrigan JM, Donaldson MS To err is human: building a safer health care system. Washington (DC): Institute of Medicine, 2000. p:17-25

2. Konh LT, Corrigan JM, Donaldson MS. Errors in health care: a leading cause of death and injury. In: Konh LT, Corrigan JM, Donaldson MS. To err is human: building a safer health care system. Washington (DC): Institute of Medicine; 2000. p 26-48.

3. Carvalho M, Vieira A. Erro médico em pacientes hospitalizados. J Pediatria 2002: 78 (4): 21-9.

4. Berlin CM, Mc Carver DG, Notterman DA, Ward RM, Weismann DN, Wilson GS, et al. Prevention of medication errors in the pediatric inpatient setting. Pediatrics 1998; 102: 428-30.

5. Carvalho VT, Cassiane SHB. Análise dos comportamentos dos profissionais de enfermagem frente aos erros na administração de medicamentos. Acta Paul Enferm 2002; 15(2): 45-53.

6. Konh LT, Corrigan JM, Donaldson MS. Why do errs happen?. In: Konh LT, Corrigan JM, Donaldson MS. To err is human: building a safer health care system. Washington (DC): Institute of Medicine; 2000. p 49-68.

7. Sawicki AM. History, legislation, and standards. In: Kunh MM. Pharmacotherapeutics. A nursing process approach. $2^{\text {nd }} \mathrm{ed}$. Philadelphia (PA): F.A. Davis Company; 1998. p. 25-48.

8. Barker B. Error rate by err or category and facility type. Arch Intern Med 2002 Sep; 16(12):1987-93.

9. National Coordinating Council for medications errors reporting and prevention. Ncc MERP taxonomy of medication errors. (online) Rockville: Ncc MERP, 1998. [cited nov 11 1998]. Available in: URL: http://nccmerp.org/tax00514.pdf.

10. Pedreira MLG, Marin HF. Patient safety initiatives in Brazil: a nursing perspective. Int J Med Inform 2004 Jul; 73: 563-7.

11. Santos SR, Paula AFA, Lima JP. O enfermeiro e sua percepção sob o sistema manual de registro no prontuário. Rev Lat-am Enfermagem 2003; 11(1): 80-7.

12. Fernandes RAQ, Salum MGL, Teixeira MB, Lemmi RCA, Miura M.
Anotações de enfermagem. Rev Esc Enferm USP 1981; 15(1): 63-8.

13. Bohony J. Common IV complications and what do about them. Am J Nurs 1993; 10: 45-9.

14. Phillips LD. Administração da terapia intravenosa. In: Philips LD. Manual de terapia intravenosa. $2^{\text {a. }}$ ed. Porto Alegre (RS): Editora Artmed; 2001. p. 301-25.

15. Barker KN, Flyn EA, Pepper GA, Bates DW, Mikael RL. Medication errors observed in 36 health care facilities. Arch Intern Med 2002; 162(2):1897-903.

16. Bohomol E. Erros de medicação: Causas e fatores determinantes sob a ótica da equipe de enfermagem [dissertação]. São Paulo (SP): Universidade Federal de São Paulo; 2002.

17. Coimbra J. Conhecimento de auxiliares de enfermagem sobre erros de medicação [tese]. Ribeirão Preto (SP): Escola de Enfermagem de Ribeirão Preto da Universidade de São Paulo; 2004.

18. Committee on the Work Environment for Nurses and Patient Safety, B. O. H. C. S. Work and workplace design to prevent and mitigate errors. In: Page AE, editors. Keeping patients safe: transforming the work environment of nurses. Washington (DC): National Academies Press; 2004. p.226-85.

19. Dreyer E. Definição operacional da qualidade da assistência de enfermagem em unidade de terapia intensiva de um hospital escola. Rev Bras Enferm 1997; 50(2): 229-46.

20. Runciman WB, Merry AF, Tito F. Error, blame, and law in health care - an antipodean perspective. Ann Intern Med 2003; 138(2): 974-9.

21. Kuhn AM, Youngberg BJ. The need for risk management to evolve and assure a culture of safety. Quality Safe Health Care 2002; 11:158-62.

22. Bates DW, Gawande WW. Patient safety: improving safety with information technology. N Eng J Med 2003; 348(25): 2526-34.

23. Van der Castle B, Kim J, Pedreira MLG, Paiva A, Gossen W, Bates DW. Information technology and patient safety in nursing practice: an international perspective. Int J Med Inform 2004; 73: 607-14.

24. Coimbra JA, Cassiane SH. Nursing responsibility in the administration of medications: some thoughts about a safe practice with care quality. Rev Lat-am Enfermagem 2001 Mar; 9(2):56-60. 